\title{
Aerodynamic Optimization of Shrouded Wind Turbines
}

\author{
A. Aranake K. Duraisamy \\ Department of Aerospace Engineering, University of Michigan, Ann Arbor, MI 48109 \\ :
}

\section{ABSTRACT}

An axisymmetric Reynolds Averaged Navier-Stokes (RANS) solver is used along with an actuator disk model for the analysis of shrouded wind turbine flow fields. Following this, an efficient blade design technique that maximizes sectional power production is developed. These two techniques are incorporated into an optimization framework that seeks to design the geometry of the shroud and rotor to extract maximum power under thrust constraints. The optimal solution is also evaluated using a full three-dimensional RANS solver, suggesting the viability of the design. The predicted optimal designs yield power augmentations well in excess of the Betz limit, even if the normalization of the power coefficient is performed with respect to the maximum shroud area. Copyright (c) 2016 John Wiley \& Sons, Ltd.

\section{KEYWORDS}

shrouded wind turbines, aerodynamic optimization

Received...

\section{INTRODUCTION}

The function of a shroud in a shrouded (or diffuser-augmented) wind turbine is to increase the velocity of the air passing through the rotor plane, thus increasing the generated power compared to an open turbine using a similar size rotor. Another important benefit of using a shroud is to reduce the cut-in speed of a wind turbine. Lower cut-in speeds mean that a turbine can remain operational during a larger part of the year, increasing the total annual production of electricity. The loads on the blade are affected in two competing ways, as the relief provided by a shorter blade in terms of bending moment is This is the author manuscript accepted for publication and has undergone full peer review but has not been through the copyediting, typesetting, pagination and proofreading process, which may leădo do difterences appear to have been first explored in the published literature by Lilley and Rainbird [19] These as doreselarcheo 0 deswibed 10 the power augmentation of shrouded turbine in terms of head loss through the diffuser. The

Prepared using weauth.cls [Version: 2010/06/17 v1.00] 
researchers determined that gains in performance were due to a decrease in tip loss of the rotor as well as the increased axial velocity. Early experimental investigations of shrouded turbines were performed in the 1980s by Gilbert and Foreman at Grumman Aerospace Corp [13, 12]. These authors used a baseline diffuser that was conical in shape at the high angle of attack of $60^{\circ}$. The turbine rotor was approximated by porous screens, which produce drag while permitting air to pass through much like a turbine. Based on a simple cost model, the authors predicted that a shrouded turbine can produce the same energy as an open turbine at half the cost. Further experimental work exploring several shroud shapes was performed by Igra [15]. This work also made use of porous screens with various drag coefficients to approximate the behavior of a turbine. Through wind tunnel testing, it was determined that the power coefficient could be improved by $80 \%$ by a shroud, with an additional $25 \%$ increase by bleeding airflow from the shroud exterior.

These studies led to the development of the first full-scale prototype of a shrouded turbine, known as the Vortec 7. This $7.3 m$ diameter turbine, which was erected in 1997, was found to underperform drastically compared to predictions from simple low-order methods [27]. The large shroud required for this turbine was supported by a bulky truss structure which was unable to yaw to face the wind. Ultimately, the shrouded turbine was deemed unprofitable and work was largely discontinued.

Werle [33] and Jamieson [16] have presented low-order theories of shrouded wind turbines that highlight the potential of these turbines to overcome the Betz limit. These theories are evaluated in Ref. [4].

Abe and Ohya $[2,1,26,25]$ have developed a so-called "flanged diffuser" concept which uses a rim on the trailing edge of the diffuser to deliberately induce separation. As a consequence of this separation, a low pressure region forms behind the shroud, drawing more mass through the interior. A custom turbulence model, developed and tuned for this purpose, is shown to provide accurate predictions of velocity and pressure profiles for shrouds [2, 1]. An experimental prototype produces 4-5 times the power of their turbine alone [26]. Shrouds of various lengths are considered, leading to the development of a $5 \mathrm{~kW}$ turbine [25]. Several of these turbine are erected for pumping water in a rural location in northern China. This group is also actively developing an offshore shrouded turbine model.

Van Bussel [32] has comprehensively reviewed the literature and concludes that if the power coefficient is normalized by the maximum shroud area, the large power coefficients reported in prior work are reduced to below the Betz limit.

In this work, an efficient blade design technique is developed and coupled to an intermediate fidelity CFD approach. This methodology is used to optimize the shroud geometry and blade properties with the objective of achieving power augmentation ratios well beyond the Betz limit. 


\section{COMPUTATIONAL METHODOLOGY}

The computations employ the OVERTURNS code [7, 11, 10,9] which performs a structured overset mesh solution of the Reynolds Averaged Navier-Stokes (RANS) equations. A Spalart Allmaras-based model is used for the closure of turbulence and transitional processes. The equations of motion are solved using a third order accurate finite volume method. Time integration is performed using the implicit Euler scheme within the approximate diagonal implicit (ADI) framework [28]. Overset connectivity is determined using an implicit hole cutting scheme developed by Lakshminarayan [17]. Extensive validations of the transition and turbulence models have been performed against experimental data by the authors in Refs. $[3,5]$. This includes two dimensional wind turbine airfoils as well as threedimensional wind turbines over a range of wind speeds. In prior work [5], detailed three dimensional simulations of shrouded turbines, using approximately 25 million grid cells required $6000 \mathrm{CPU}$ hours per run. Such calculations reveal many physical features, but their expense and complexity makes them inappropriate in a design setting, when hundreds of evaluations are required and a smooth design space is desired.

A considerable reduction in cost can be achieved by substituting the three-dimensional RANS simulation with an axisymmetric model. The equations governing axisymmetric flow are derived by beginning with the cylindrical form of the governing equations and eliminating terms associated with derivatives in the azimuthal or "swirl" direction. The axisymmetric solver accounts for the shroud and the rotor is represented by including an actuator disk model [4]. At each iteration of the flow solver, the sectional lift and drag are computed using the local velocity and tabulated experimental data [29] with known twist and chord distributions. Additional losses due to three-dimensionality are accounted for using Prandtl's tip correction (refer Equation 2). Thrust and power can then be computed by integrating the forces over the disk and are introduced into the momentum and energy equations as source terms.

\subsection{Verification and Validation}

In this work, as a first verification study of the implementation of the axisymmetric terms, the solver is compared against the results of Bontempo et al. [8]. In that work, a semi-analytical method was derived to compute the solution to the axisymmetric potential flow equations for ducted propellors and turbines using an actuator disk model. This was

accomplished using a Green's function approach. One difficulty with this method is that it requires the specification of the wind turbine load as a function of the stream function $\psi$, which is not known a priori. In Ref. [8] computed results are presented which make it possible to determine the load as a function of radial location, thus providing a convenient solution for verification. The turbine is represented by an actuator disk across which there is a distributed pressure drop as discussed above. A NACA5415 airfoil is used for the shroud geometry with dimensions chosen to match those in 

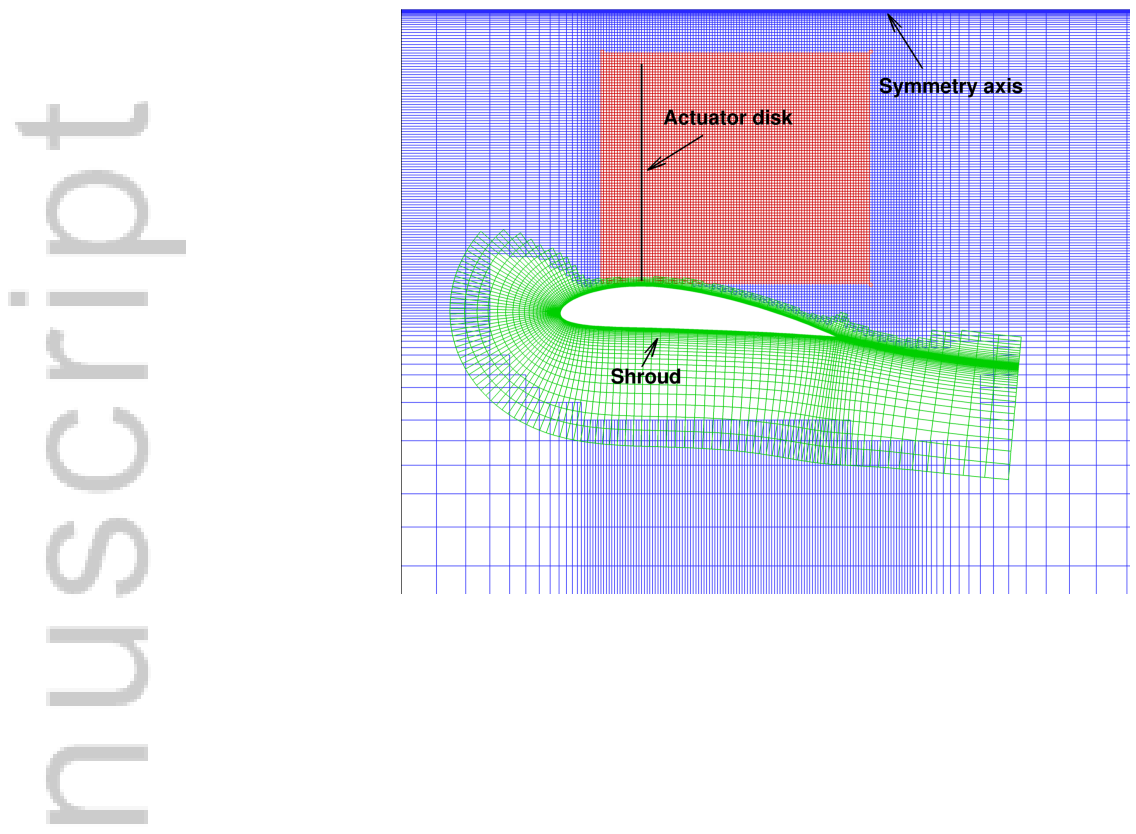

Figure 1. Near-field grid used for verification of BET method against potential flow solution given in [8]
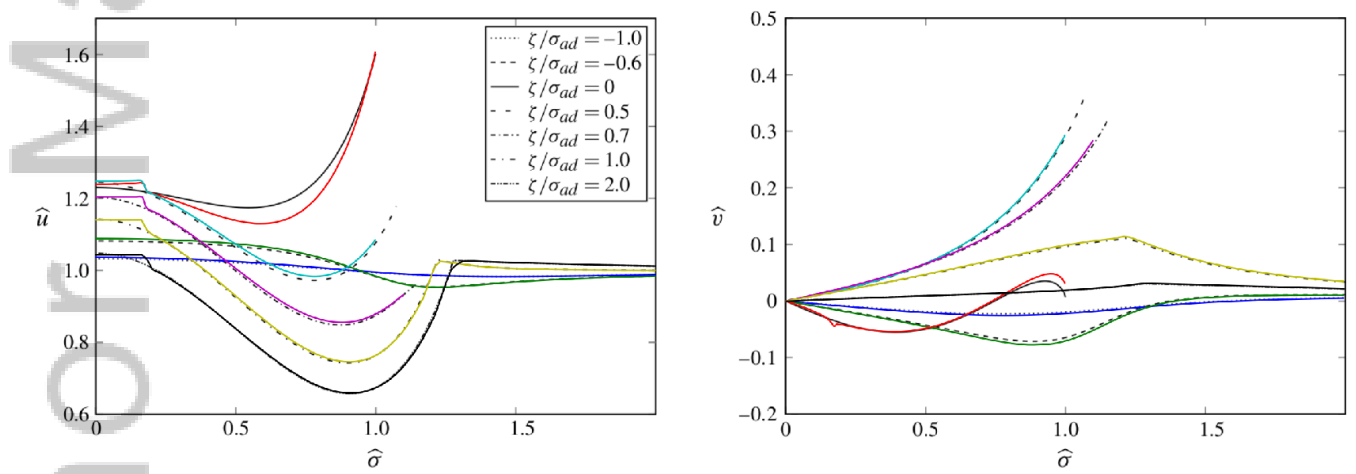

Figure 2. Velocity profiles at several axial locations. Verification of axisymmetric solution (colored lines) against [8] (black lines).

[8]. The grid used for this calculation is shown in Figure 1. Velocities profiles from this calculation are shown in Figure 2. Following the notation of the authors, the velocity is represented as $\hat{u}=\frac{u}{U_{\infty}}$ and $\hat{v}=\frac{v}{U_{\infty}}$. The radial coordinate is $\hat{\sigma}=\frac{r}{R}$. The quantities are shown for several axial locations $\zeta$. The results agree quite well. A small discrepancy is seen for $\hat{\sigma}<0.2$ which is due to a clipping of the grid near the symmetry axis as seen in Figure 1. This result provides confidence in the accuracy of the implementation of the axisymmetric terms.

In addition to this verification, validation of the axisymmetric solver was performed by comparison against the results of a set of 3D RANS solutions of a shrouded wind turbine (the details of these three-dimensional computations are discussed 

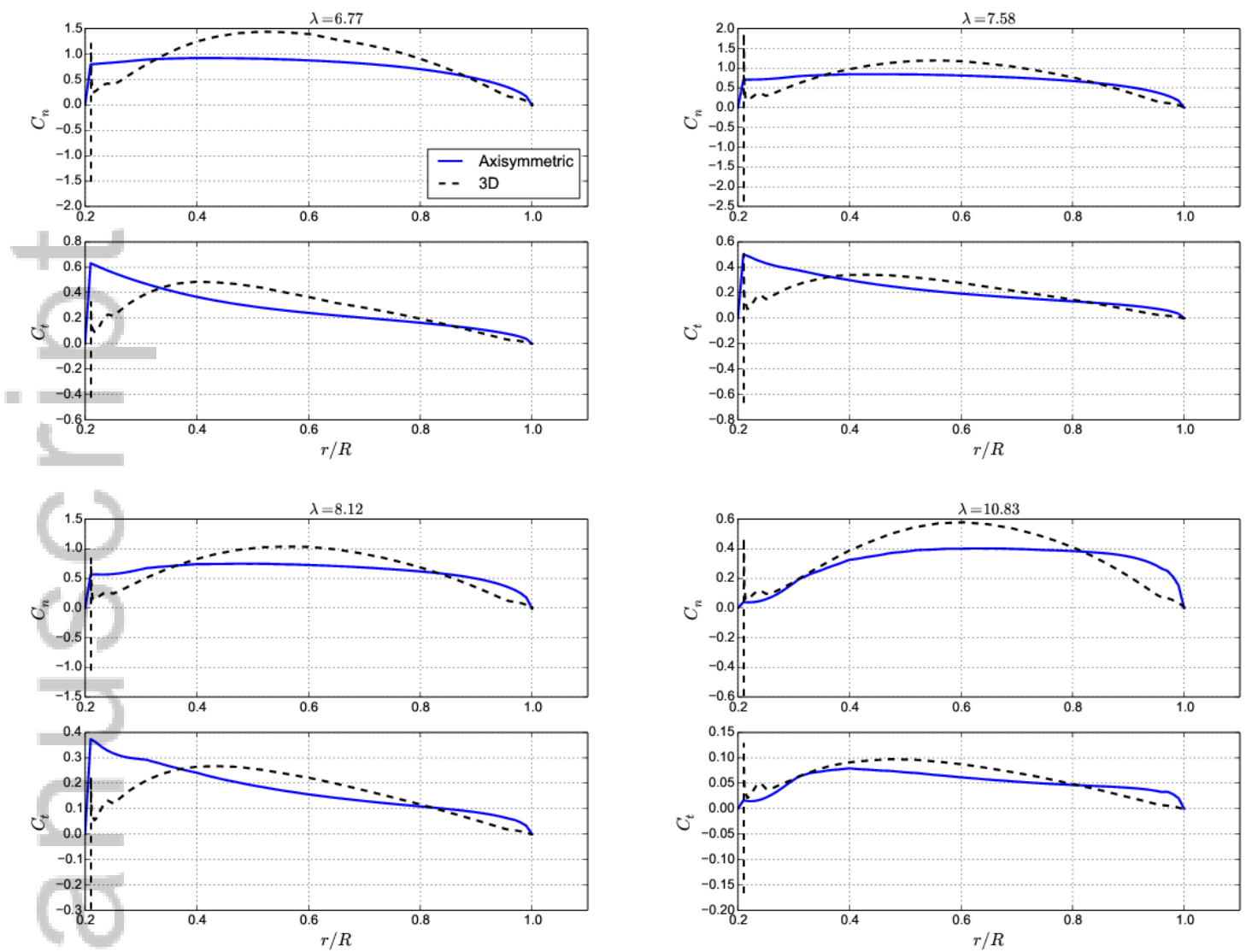

Figure 3. Spanwise load distributions at different tip speed ratios $\lambda$. Verification of axisymmetric solver against 3D

in Ref. [5]. Figure 3 shows the load distributions for shrouded turbine calculations performed using both the axisymmetric and 3D solvers. Considering the complexity of the shrouded turbine flowfield, the results are seen to agree reasonably well. While the correlation is not exact, it lends confidence that the assumptions involved in the axisymmetric calculations are reasonable for engineering purposes.

\section{DESIGN METHODOLOGY}

The process of designing a system as complicated as a wind turbine is inherently multi-disciplinary in nature. Approaches to (Multi-disciplinary optimization) MDO are broadly divided into two categories [20]:

- Monolithic - All of the design variables are considered simultaneously, and a single optimization problem is solved.

- Distributed - The design variables are divided, usually into their respective disciplines. Optimizations are performed for each discipline, and information is communicated between disciplines by a high-level architecture. 
Recent application to wind turbines [23] compares a monolithic approach to a particular distributed approach and finds that the former leads to a superior optimal design by about $2 \%$ in terms of annual cost of energy (ACEP) for a particular set of methodologies. This difference is significant for utility-scale planning, where small percentage differences in the power coefficients in individual turbines lead to considerable differences in total dimensional watts generated. Still, distributed optimization methods are often preferred. Such methods permit specialists in each of the various disciplines to concentrate their effort on the components they know best and work in parallel to approach an aggregate solution. This approach is more flexible than a monolithic approach, as each discipline can select the level of modeling fidelity most appropriate to the situation and maximize its own efficiency. It is typical of practical MDO problems for the requirements of the different disciplines to compete with one another. A classic example from aeronautics is the optimal design of an airplane wing. Aerodynamic considerations alone lead to a high aspect ratio wing with an elliptical load distributions, whereas structural considerations favor a short blocky wing. When the disciplines are considered together, the resulting wing is not optimal with regard to either discipline, but rather balances the needs of each to minimize cost.

In this section, a method for the aerodynamic optimization of wind turbines is presented. The utility of this model is two-fold. First, it can serve as the aerodynamics component of a larger MDO framework. Second, it explores the design space to provide insight into the theoretical capabilities of a shrouded turbine.

\subsection{Inverse BET method}

This section considers the design of the twist distribution of a wind turbine blade for operation for a shrouded turbine. A variation of the Blade Element Theory (BET) method is developed and will be referred to as the inverse BET method. This method serves as a convenient tool for determining the optimal blade twist and is an original contribution.

Modern turbines operate at variable speeds, and so development of a single optimal design for ACEP requires knowledge of how much time the turbine spends operating at each tip speed ratio. In practice, this depends on many factors (site conditions, gearbox, etc.) and will vary between turbines and sites. Modern turbines permit the use of variable rotation rates and employ advanced control systems to maintain an efficient tip speed ratio during normal operation. If it is satisfactory to assume that the turbine will spend much of its operating time at a single $\lambda$, then it is sufficient to optimize the $C_{P}$ in lieu of ACEP. In this case, the inverse BET model provides a convenient way to solve for the optimal blade twist distribution. The blade tangential force coefficient is computed by

$$
C_{t, i}=\left(C_{l, i} \sin \left(\phi_{i}\right)-C_{d, i} \cos \left(\phi_{i}\right)\right) F_{i}
$$

where $i=1, \ldots, n$ and $n$ is the number of annular rings used to discretize the turbine. This coefficient, which is based on the local flow angle $\phi$, represents the turbine driving force, which is responsible for power conversion. The factor $F$ is the 
Prandtl tip loss factor, which approximates the 3-dimensional effect of the tip vortex. It is given by

$$
F_{i}=\frac{2}{\pi} \cos ^{-1}\left[e^{-\frac{B\left(R-r_{i}\right)}{2 r_{i} \sin \phi_{i}}}\right]
$$

where $B$ is the number of blades.

To make use of tabulated airfoil data, the force coefficients $C_{l}$ and $C_{d}$ are normalized with respect to the local velocity. Computing the power coefficient, which is based on freestream values, requires the following renormalization of this coefficient.

yorent.

$$
\begin{aligned}
\widetilde{C_{t, i}} & =C_{t, i} \frac{\frac{1}{2} \rho_{i}\left(u_{z, i}^{2}+\left(\omega r_{i}\right)^{2}\right) c_{i} \Delta r_{i}}{\frac{1}{2} \rho U_{\infty}^{2} \pi R^{2}} \\
& =C_{t, i}\left(\tan ^{2}\left(\phi_{i}\right)+1\right)\left(\lambda \frac{r_{i}}{R}\right)^{2} \frac{c_{i} \Delta r_{i}}{\pi R^{2}}
\end{aligned}
$$

Isolating the dependence on $\phi$ the following definition

$$
f(\phi)=C_{t}(\phi)\left(\tan ^{2}(\phi)+1\right)
$$

is used. In the standard BET approach, the angle of attack is computed using the twist and flow angle, $\alpha=\phi-\theta$. In the inverse BET method, this process is reversed. Rather than using a prescribed blade twist, the angle of attack $\alpha$ is selected to maximize Equation 5. This is a one-dimensional optimization problem and is solved using the Golden section method [21].

Figure 4 shows optimal values for $f(\phi)$ assuming a Prandtl tip correction of $F=1$. The angle of attack is bracketed such that $\alpha \in\left[0^{\circ}, 12^{\circ}\right]$, avoiding airfoil stall. As the flow angle $\phi$ increases, the optimal angle of attack also increases until it reaches the bracket boundary of $\alpha=12$. Once the optimal $\alpha$ is known, the twist is selected to achieve this angle of attack. By inverting the BET method in this way, an optimal blade twist distribution is designed as the CFD solver converges. This procedure is an instance of the "all-at-once" paradigm for optimization problems.

To verify convergence of the inverse BET method, the procedure was applied to the NREL Phase VI blade at $\lambda=6$. For this test case, the L2-norm of the difference in twist distribution between successive iterations was verified to converge to machine precision, indicating that upon CFD convergence the inverse BET method also converges to a unique twist distribution. An initial implementation of this technique approximated the airfoil lift and drag curves using piecewise linear approximations. With this approach, small oscillations of the twist distribution persisted after many iterations and interfered with the convergence of the CFD solver. This problem was remedied by replacing the piecewise linear approximation with a cubic spline representation, which has continuous first derivatives.

Wind Energ. 2016; 00:1-19 (C) 2016 John Wiley \& Sons, Ltd. 

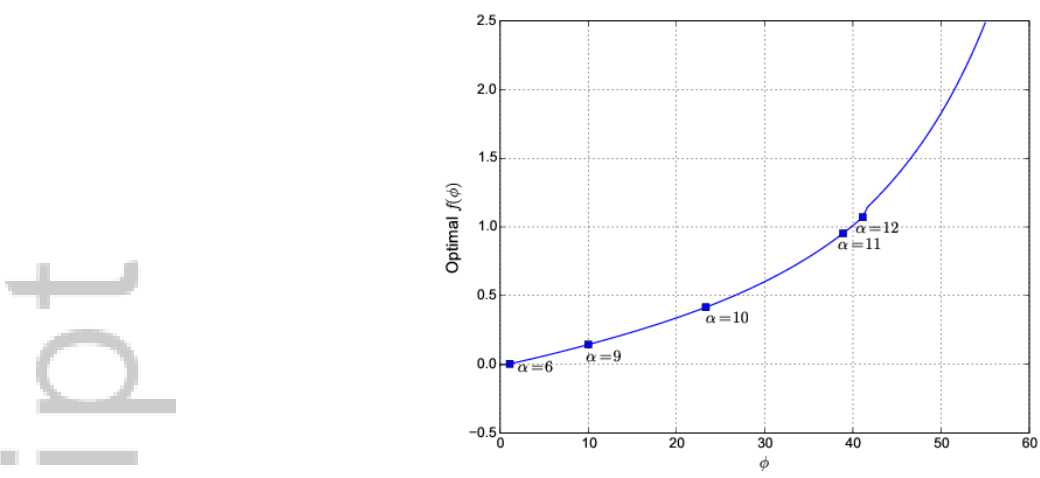

Figure 4. Optimal values of $f(\phi)$ assuming a Prandtl tip loss of $F=1$. The optimal angle of attack $\alpha$ increases until it reaches the bracket endpoint of $\alpha=12$ and remains constant thereafter.

\section{OPTIMIZATION}

This section describes a procedure for shrouded turbine optimization, in which the blade twist is considered along with the shape of the shroud. The geometry of the shroud is parameterized using the PARSEC variables [30].

The PARSEC family of design variables permit the representation of a large family of airfoils using 11 basic parameters. The upper and lower surfaces of the airfoil are each defined by 6 geometric parameters. The upper and lower curves are then each defined as a combination of basis functions, as in

$$
\begin{aligned}
& z_{\text {upper }}=\sum_{i=1}^{6} a_{i} \psi_{i}(x) \\
& z_{\text {lower }}=\sum_{i=1}^{6} b_{i} \psi_{i}(x) .
\end{aligned}
$$

Determining coefficients $a_{i}$ and $b_{i}$ from geometric parameters requires a matrix inversion for each surface. The choice of basis functions $\psi_{i}$ is arbitrary provided that the functions are orthogonoal, $\psi$ is twice differentiable, and $\psi(0)=\psi(1)=0$.

In the present work, the functions used are

$$
\psi_{i}=\sin \left(\frac{i x}{2 \pi}\right)
$$

which makes Equations 6 and 7 equivalent to truncated Fourier series. A more common choice of basis functions for the PARSEC algorithm in literature is a family of polynomials, but the above representation was found to admit a slightly better approximation of the S1223 airfoil *, which is used as the initial condition for the optimizations that follow. An implementation of the PARSEC algorithm was written in the Python language using the SciPy package. The algorithm, which involves the solution of two systems of six equations each, requires very little computer time compared to a CFD

* The S1223 is a high lift airfoil, which was found to offer good power augmentations in prior work [5] 
Table I. Description of PARSEC variables and initial values

\begin{tabular}{|c|c|c|}
\hline Variable & Description & Initial value \\
\hline$r_{\text {leup }}$ & Radius of curvature of upper surface at leading edge & 0.12911 \\
\hline$r_{l e l o}$ & Radius of curvature of lower surface at leading edge & 0.29947 \\
\hline$X_{u p}$ & $x$-coordinate of extremum in upper surface & 0.04508 \\
\hline$X_{l o}$ & $x$-coordinate of extremum in lower surface & 0.31139 \\
\hline$Z_{u p}$ & $z$-coordinate of extremum in upper surface & 0.01762 \\
\hline$Z_{l o}$ & $z$-coordinate of extremum in lower surface & 0.13640 \\
\hline$Z_{\text {xxup }}$ & $z^{\prime \prime}$ at extremum in upper surface & 0.01839 \\
\hline$Z_{x x l o}$ & $z^{\prime \prime}$ at extremum in lower surface & 0.00870 \\
\hline$\beta_{t e}$ & Angle between surfaces at trailing edge & 0.08903 \\
\hline$\alpha_{t e}$ & Angle between trailing edge and axis & 0.51990 \\
\hline$Z_{t e}$ & $z$-offset of trailing edge & 0.0 \\
\hline$\Delta Z_{t e}$ & Trailing edge thickness & 0.0 \\
\hline
\end{tabular}

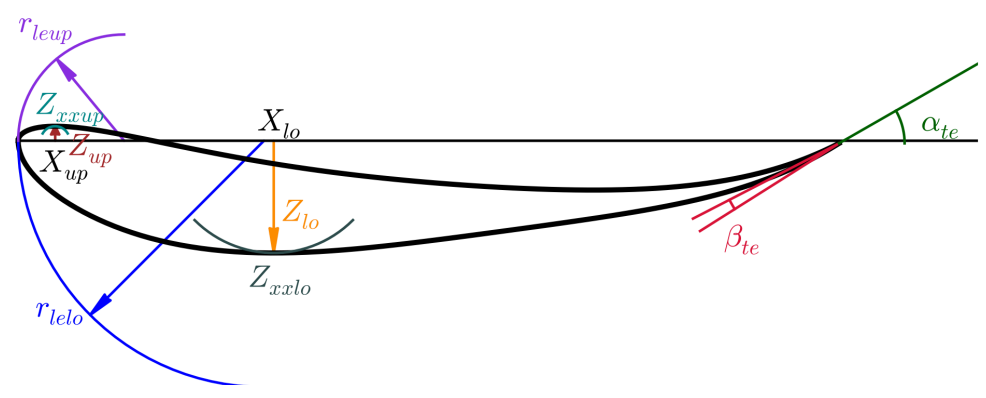

Figure 5. PARSEC parameterization approximating $\$ 1223$ airfoil.

solution. Figure 5 shows the geometric variables used for this approximation. A description of each variable along with its initial value is given in Table I.

The optimization problem is formulated as follows:

$$
\begin{array}{ll}
\underset{\vec{\alpha}}{\operatorname{minimize}} & \mathcal{J}(\vec{\alpha}) \\
\text { subject to } & t_{\min } \leq t_{j} \leq t_{\max }, j=1, \ldots, m
\end{array}
$$

where the vector $\vec{\alpha}$ consists of the design variables. The last two PARSEC variables, $Z_{t e}$ and $\Delta Z_{t e}$, are not included in $\vec{\alpha}$. Instead, varying the $z$ coordinate of the trailing edge is controlled by including the angle of attack $\alpha$ as a design variable. The trailing edge thickness is forced to remain zero. In addition to these geometric quantities, the tip speed ratio $\lambda$ is also 


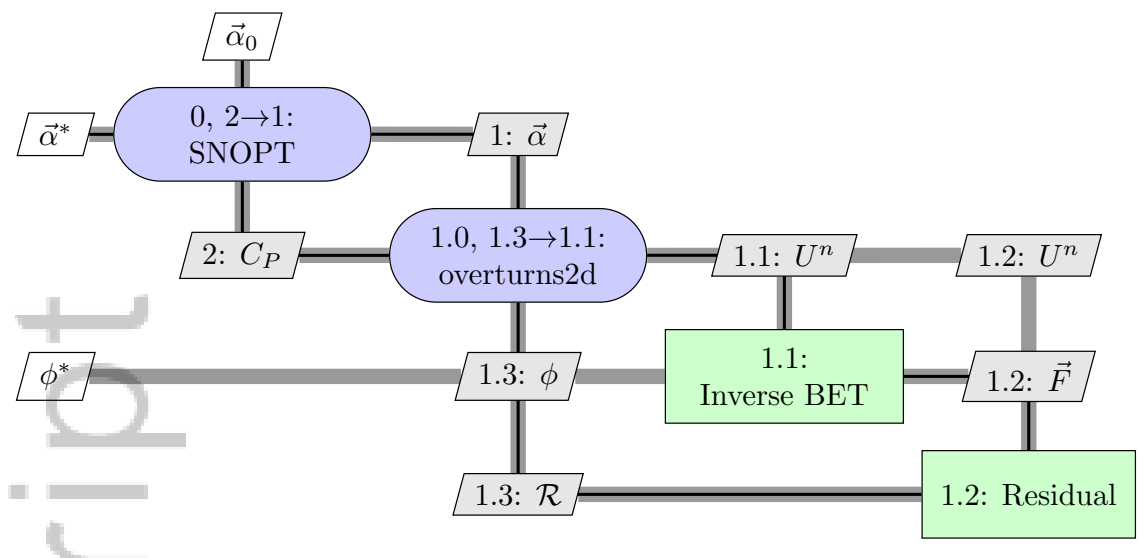

Figure 6. XDSM chart of combined shroud and blade twist optimization

included as a design variable. Hence the 12 component vector $\vec{\alpha}$ consists of the first ten PARSEC variables along with the shroud angle of attack and tip speed ratio. The objective function $\mathcal{J}$, to be minimized, is a renormalized power coefficient defined by

$$
\mathcal{J}=-C_{P} \frac{A}{A_{\max }}+p\left(C_{T}\right)
$$

where $A_{\max }$ is the maximum cross-sectional area of the shroud. In practice, $A_{\max }=\pi r_{\mathrm{te}}^{2}$ where $r_{\mathrm{te}}$ is the radial coordinate of the shroud trailing edge. Given a set of PARSEC variables, the upper and lower surfaces are constructed at $m=100$ points in the chordwise direction distributed using the Chebyshev collocation points. The thickness at each of these points is $t_{j}=z_{\mathrm{upper}, \mathrm{j}}-z_{\text {lower }, \mathrm{j}}$ and is constrained above and below by $t_{\max }=0.2$ and $t_{\min }=0$ respectively. An Extended Design Structure Matrix (XDSM) chart [18], shown in Figure 6, visualizes the data flow observed in this process.

The function $p\left(C_{T}\right)$ is a function included to penalize high values of thrust. Without a penalty for high thrust, the optimizer increases loading to the point where the turbine enters a vortex ring state. This commonly occurs around $C_{T}=1.1$ for open turbines [31] and was found to occur at about the same loading for shrouded turbines. In practice, such high loadings are undesirable as they are not robust to increased windspeeds. Furthermore, increased thrust coefficients lead to excessive structural tower cost [22]. The function selected for the present work is

$$
p\left(C_{T}\right)= \begin{cases}0 & : C_{T}<C_{T \max } \\ 100\left(C_{T}-C_{T \max }\right)^{4} & : C_{T} \geq C_{T \max }\end{cases}
$$

where, $C_{T \max }=0.7$. Optimizing for power with this penalty function effectively sets the thrust coefficient to $C_{T}=$ $C_{T \max }$ with leniency which can be tuned by adjusting the coefficient of 100 . Thrusts as high as $C_{T}=1.1$ are effectively prohibited. In a multi-disciplinary setting, the value of $C_{T \max }$ can be adjusted according to structural needs. 
The optimizer selected for shroud geometry is the Sparse Nonlinear OPTimizer (SNOPT) [14], which implements a sparse sequential quadratic programming (SQP) algorithm. At each iteration until convergence, this type of optimizer solves a quadratic optimization problem to determine an appropriate search direction followed by a line search in that direction.

Simulatenous optimization of the shroud geometry and blade twist proceeds as follows. An initial guess of the design variables, $\overrightarrow{\alpha_{0}}$, is passed to the optimizer. The Python implementation of PARSEC is then used to generate the upper and lower surfaces of the airfoil, each of which are defined by $m=100$ points following a Chebyshev distribution. Next, these In

coordinates are passed to a hyperbolic grid generator which creates a structured C-mesh about the airfoil. This mesh is then passed through a preprocessing code which scales, rotates, and translates the shroud into the proper location before joining it to the background and blade meshes. The solver overturns $2 \mathrm{~d}$ is then executed, and the design is evaluated. This procedure is described schematically in Figure 6. It bears mentioning that this type of nested optimization is not guaranteed to lead to a globally optimal solution. In other words, the use of a single optimizer to manage both the twist distribution and the shroud airfoil geometry may lead to an even better final design. The outlined procedure is, however, computationally efficient, and as will be shown it leads to a significant improvement in the objective function.

Gradients are computed using finite-differences of each of the design variables. Finite-differences for gradients of the objective function require an additional flow solution per variable. To save wall time, the optimization framework was developed to compute each objective and its perturbed gradient computations simultaneously in parallel. In this way, an evaluation of a shrouded turbine and its gradients requires about 20 minutes of real time on a 12-core desktop workstation. Gradients of the thickness constraints are also performed using finite-differencing. These $m$ gradients are rapidly evaluated in serial on a single processor.

\subsection{Optimal result}

The reduction in the objective function $\mathcal{J}$ as a function of design iterations is shown in Figure 7. An improvement of $27.5 \%$ in objective is achieved. In total, 64 designs were evaluated by the optimizer. The designs plotted in Figure 7 correspond to each set of design variables $\vec{\alpha}$ attempted by the optimizer. In some cases, such as designs 10 and 15 , this design vector resulted in an airfoil shape which violated the thickness constraints. In practice, this was always caused by a self-intersecting airfoil, which violates the constraint that the thickness is everywhere greater than zero. Upon convergence, SNOPT reported that it is "unable to proceed into undefined region," which indicates that "At the final point, it appears that the only way to decrease the merit function is to move into a region where the problem functions are not defined [14]."

The initial and final shroud shapes are depicted in Figure 8. The thickness of the optimized airfoil towards the trailing edge is reduced significantly, and the minimum thickness constraint is active in this region. Trailing edge separation 

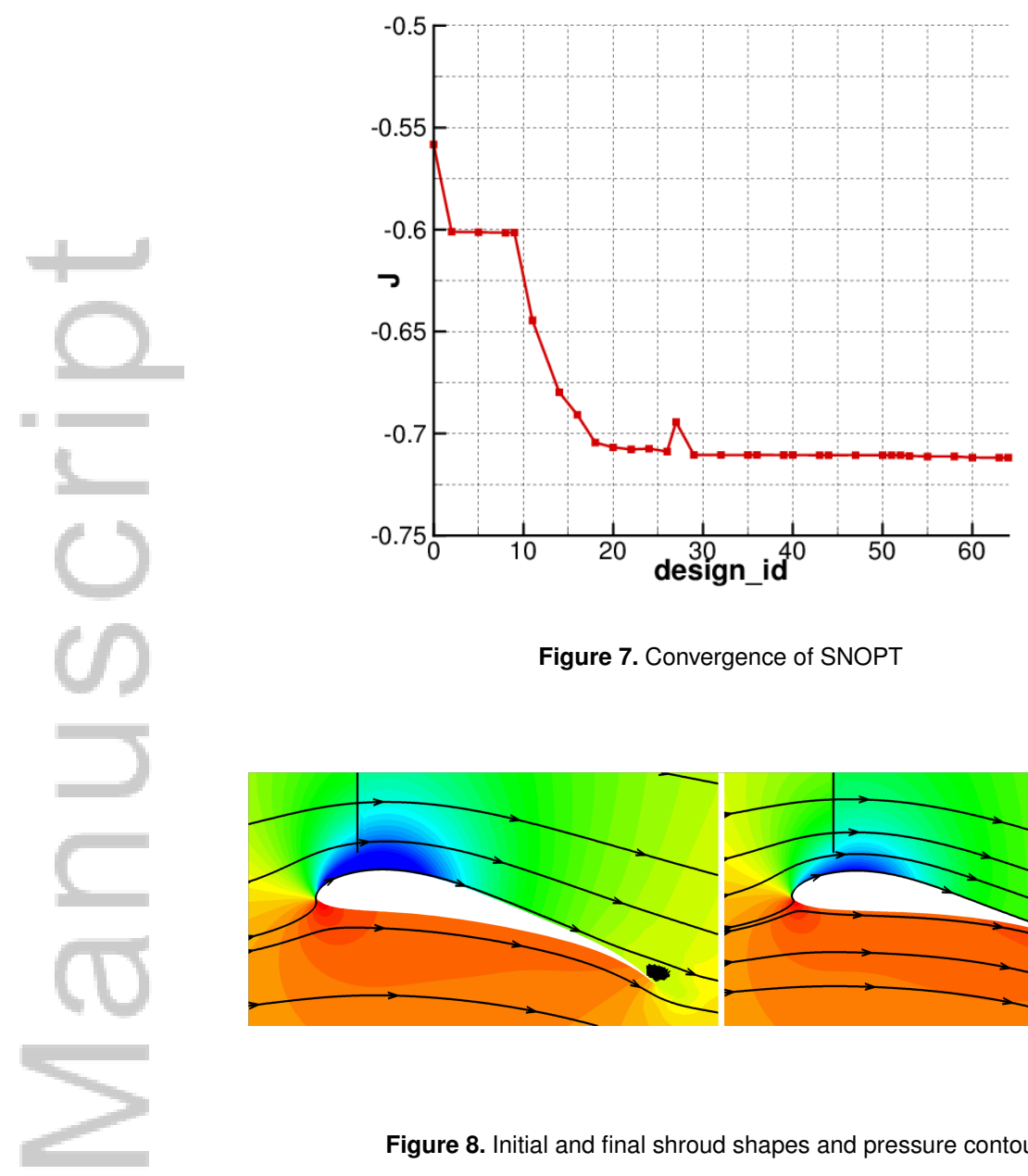

Figure 7. Convergence of SNOPT

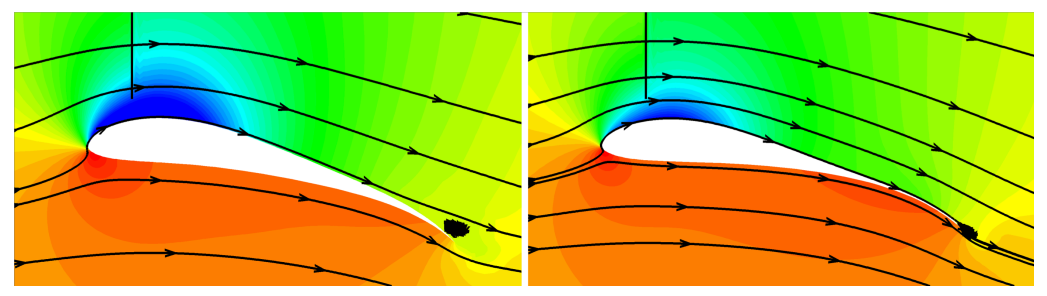

Figure 8. Initial and final shroud shapes and pressure contours

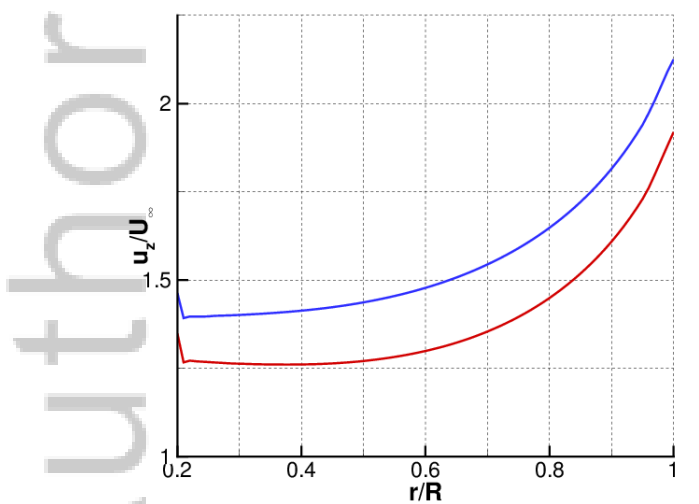

(a) Axial velocity distribution at rotor plane for initial and final design

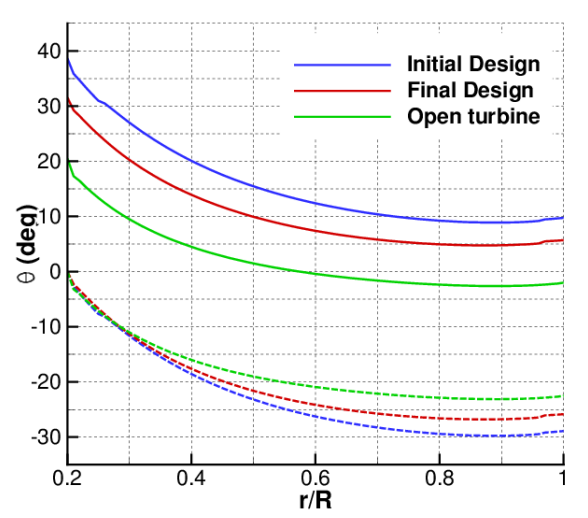

(b) Initial and final rotor twist distributions

Figure 9. Initial and optimal solutions 
Table II. Design variables and performance quantities for initial and final designs

\begin{tabular}{|c|c|c|}
\hline Variable & Initial value & Final value \\
\hline$r_{\text {leup }}$ & 0.12911 & 0.12854 \\
\hline lelo & 0.29947 & 0.28907 \\
\hline$u p$ & 0.04508 & 0.04521 \\
\hline$X_{l o}$ & 0.31139 & 0.32426 \\
\hline$Z_{u p}$ & 0.01762 & 0.01772 \\
\hline lo & 0.13640 & 0.13823 \\
\hline rxup & 0.01839 & 0.01887 \\
\hline clo & 0.00870 & 0.00849 \\
\hline$\beta_{t e}$ & 0.08903 & 0.07295 \\
\hline$\alpha_{t e}$ & 0.51990 & 0.54287 \\
\hline$\alpha$ & 15.0 & 16.52281 \\
\hline$\lambda$ & 6.0 & 7.09633 \\
\hline
\end{tabular}

\begin{tabular}{c|c|c}
\hline Quantity & Initial value & Final value \\
\hline $\mathcal{J}$ & 0.55831 & 0.71176 \\
$A_{\max } / A$ & 1.89929 & 1.85474 \\
$C_{P}$ & 1.060416 & 1.322566 \\
$r$ & 1.78945 & 2.23183 \\
$r_{r}$ & 0.94215 & 1.201095 \\
$C_{T}$ & 0.53115 & 0.75527 \\
$p\left(C_{T}\right)$ & 0.0 & 0.00093 \\
$C_{z}$ & 0.29927 & 0.39682 \\
$C_{r}$ & 3.20924 & 2.81502 \\
\hline
\end{tabular}

is present, though the size of the region of trailing edge separation is reduced considerably in the optimized design. Additionally, while leading edge loading is reduced, trailing edge loading is increased in the optimized design. In this way, some radial force is retained while the magnitude of the suction peak is attenuated. This solution is reasonable, as the optimizer tends to a very thin trailing edge which is known to be desirable for high-lift airfoils. The possibility remains, nonetheless, that the solution obtained is a local optimum and not a global one.

The initial and final twist distributions are shown in Figure 9b. For comparison, the inverse BET method was also run for an open turbine configuration at a matching windspeed and tipspeed ratio. In order to make the difference in blade shape visible, the dashed lines show each twist distribution offset by a collective angle $\theta_{0}$ such that the twist at $r / R=0$ is 0 . Note that all distributions shown are optimized twist distributions for their respective conditions. The reduction of the twist angle in the final design corresponds to a decrease in the axial flow velocity at the rotor plane.

Values of the design variables and resulting performance quantities are tabulated in Table II. The optimized result reduces the objective function $\mathcal{J}$ by both increasing the power coefficient by $24.7 \%$ and reducing the max area by $2.3 \%$. Perhaps surprisingly, the increase of velocity in the axial direction at the turbine plane, shown in Figure 9a, is reduced at the rotor plane is reduced in the optimal design. This is consistent with the reduction of radial force. The tip speed ratio of the final design is increased, leading to a net increase in velocity magnitude at the rotor plane. 


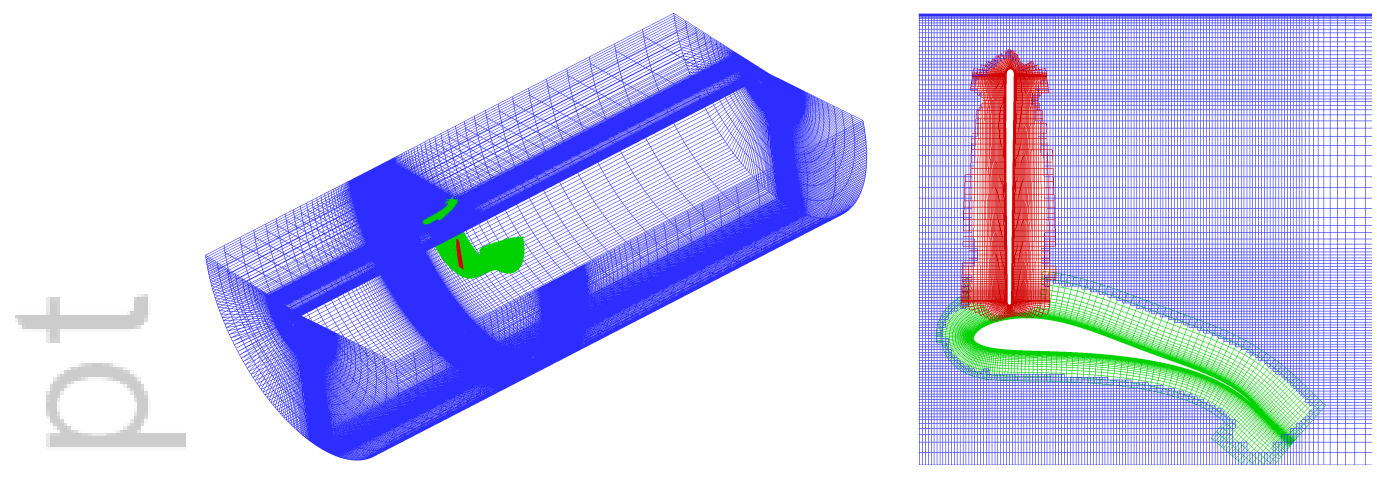

Figure 10. Grids used for 3D validation.

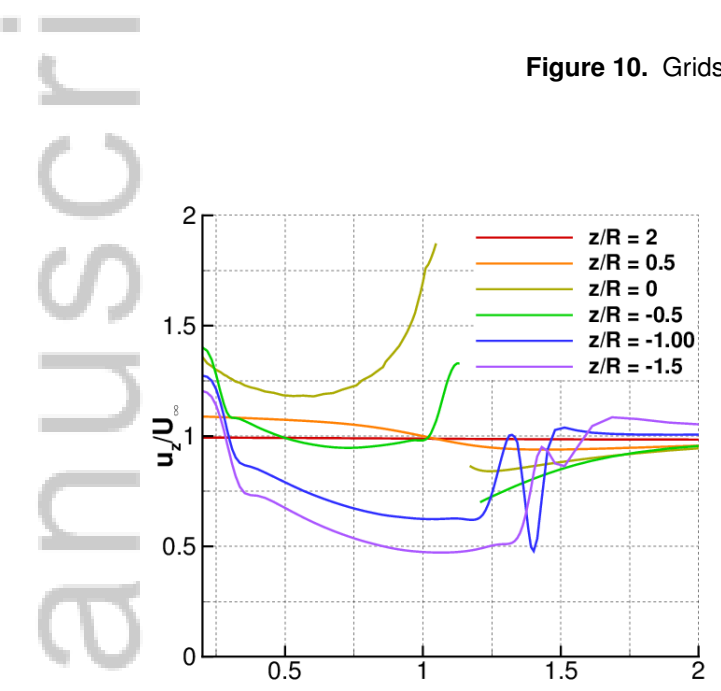

(a) Averaged velocity profiles of optimized design.

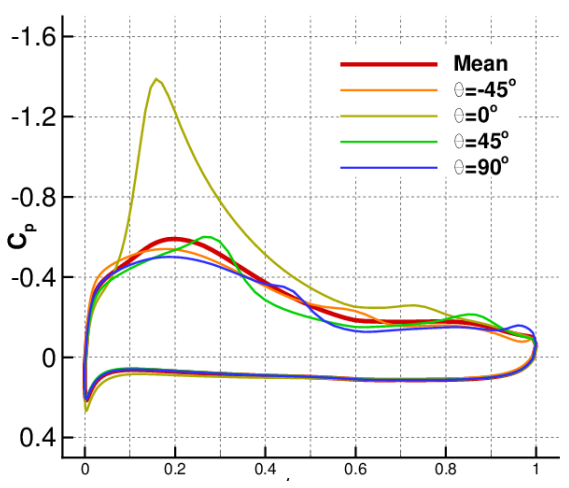

(b) Pressure coefficient along optimized shroud.

Figure 11. Velocity and pressure profiles for optimized result

\section{3D VALIDATION OF OPTIMIZED RESULT}

Validation of the optimized result of the previous section is performed by a 3D RANS calculation of wind turbine performance. As in Ref. [5], three overset meshes are used for the calculation. The blade mesh has dimensions $251 \times 101 \times 51$ in the wrap-around, spanwise, and normal directions respectively. In the same order, the shroud mesh has dimensions $217 \times 201 \times 49$. The background mesh has dimensions $201 \times 129 \times 205$ in the azimuthal, radial, and axial directions respectively. A view of the grids is shown in Figure 10.

A comparison between results from the axisymmetric solver and the full $3 \mathrm{D}$ calculation is shown in Table III. The axisymmetric optimization procedure has led to a greater power coefficient and augmentation ratio. Averaged velocity profiles for the optimized result are shown in Figure 11a. Considerable flow augmentation is present at the turbine plane which is located at $z / R=0$ as was observed in Figure 9a. The pressure coefficient at various azimuthal points along the shroud is depicted in Figure 11b. 


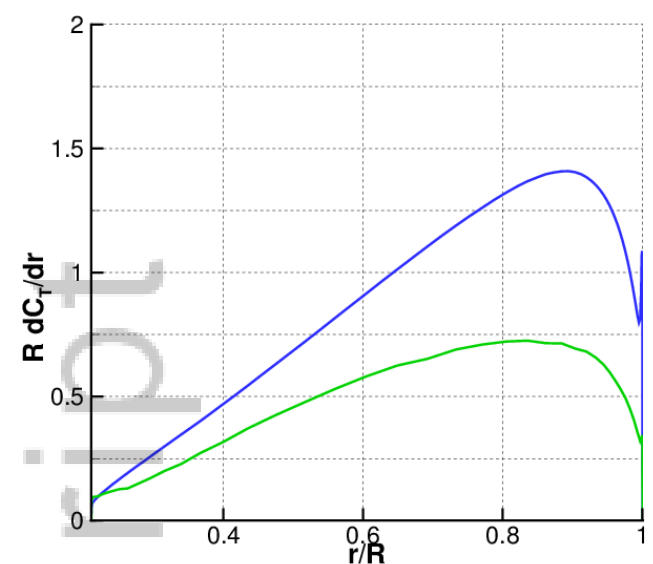

(a) Spanwise contribution to thrust coefficient

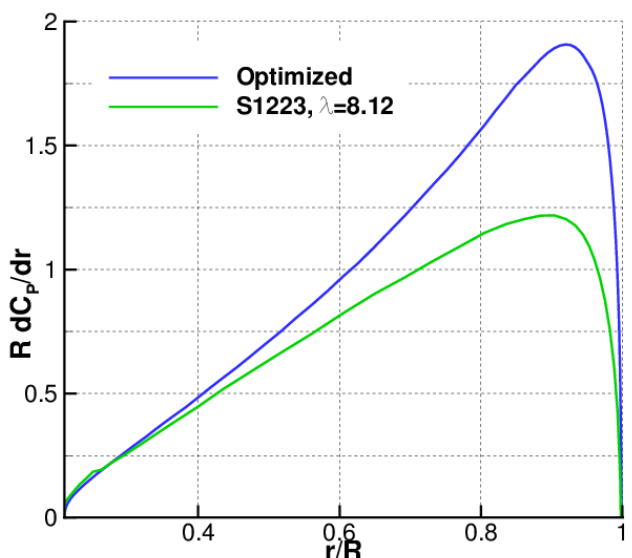

(b) Spanwise contribution to power coefficient

Figure 12. Comparison between optimized result and best performing $\$ 1223$ result

Table III. Comparison of performance parameters from axisymmetric and 3D solver

\begin{tabular}{c|c|c}
\hline Quantity & Axisymmetric solver & 3D RANS \\
\hline$C_{P}$ & 1.322566 & 1.56742 \\
$r$ & 2.23183 & 2.64502 \\
$r_{r}$ & 1.201095 & 1.42609 \\
$C_{T}$ & 0.75527 & 0.91844 \\
\hline
\end{tabular}

Figure 12 shows the spanwise thrust and power contributions along the optimized blade. These values are shown alongside the best values obtained in a prior publication [5] which used the original NREL Phase VI blade with the $\mathrm{S} 1223$ shroud. The windspeed for this case was $U_{\infty}=7 \mathrm{~m} / \mathrm{s}$ and the tip speed ratio was $\lambda=8.12$. A considerable increase in the contribution to both forces is seen across the entire blade. Towards the tip, significantly more power is generated by the optimized blade. This is because the flow acceleration is greater nearer to the shroud. The NREL Phase VI blade, which was designed to operate as an open turbine, was operating furthest from its design conditions in this region when the S1223 shroud was added. As such, there was more room for improvement at this location. The optimized result balances the nonuniformity of the flow augmentation.

A summary of results obtained from calculations in three dimensions is shown in Table IV. For each case, the trial which resulted in the greatest power coefficient is listed. Between the S1223 shroud airfoil and the optimized case, an improvement in renormalized augmentation ratio $r_{r}$ of $42 \%$ is realized. This is a very significant increase in power production which indicates the great potential for power augmentation through optimization of shrouded turbines. 
Table IV. Maximum power coefficient results for various shroud geometries in 3D

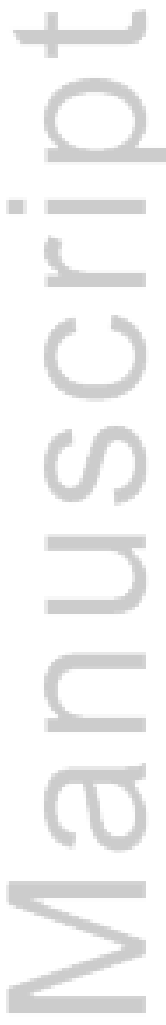

\begin{tabular}{c|c|c|c|c}
\hline Quantity & Open & NACA0006 & S1223 & Optimized \\
\hline$\lambda$ & 6.77 & 7.85 & 8.12 & 7.10 \\
$C_{P}$ & 0.38 & 0.65 & 1.15 & 1.57 \\
$r$ & 0.64 & 1.1 & 1.95 & 2.65 \\
$r_{r}$ & - & 0.63 & 1.01 & 1.43 \\
$C_{T}$ & 0.54 & 0.39 & 1.08 & 1.35 \\
\hline
\end{tabular}

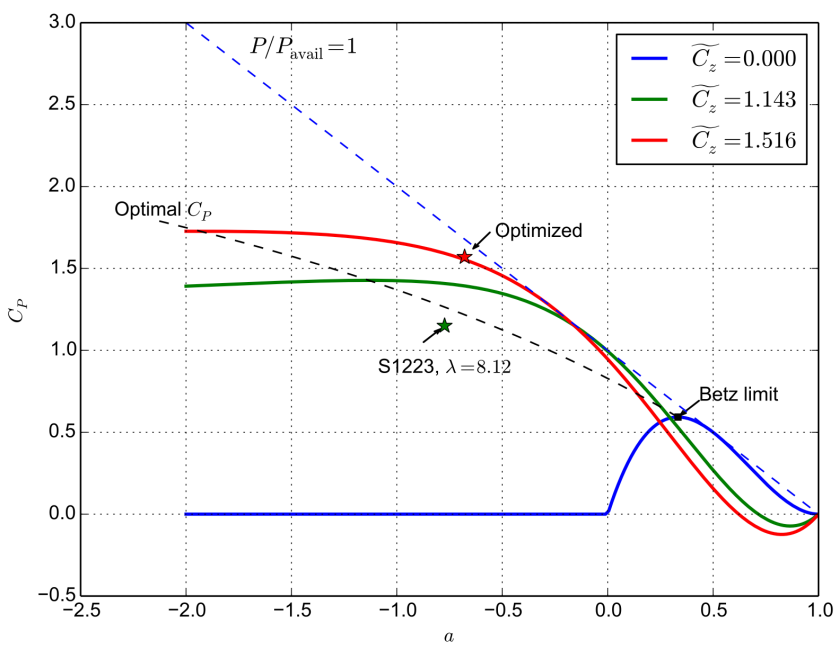

Figure 13. Location of current results with reference to momentum theory.

These two 3D runs are contextualized in Figure 13 in terms of a low-order momentum theory developed in Ref. [6]. A power increase is realized between the $\mathrm{S} 1223$ shroud and the optimized case. The axial induction ${ }^{\dagger} a$, however, is reduced in magnitude. This is consistent with the decrease in lift force between the original and final design. Rather than increase the fluid velocity at the rotor plane, the strategy of the optimizer is to increase the shroud force coefficient ${ }^{\ddagger} \widetilde{C_{z}}$. To develop a shroud which moves towards the optimal curve, the magnitude of induction must be further increased. This would require a greater lift force provided by the shroud airfoil. The magnitude of lift attainable is clearly bounded by flow separation, and the S1223 airfoil was specifically designed to test this bound. Considering this, further advances due to increasing lift force are unlikely.

$\dagger_{\text {which is }}$ the degree of deceleration of the freestream flow at the rotor disk

${ }_{\text {which }}$ is the non-dimensional axial force on the shroud 


\section{SUMMARY}

The addition of a shroud to a wind turbine can augment the mass flow through the rotor disk, increasing the power available for conversion. As an intermediate fidelity model, an axisymmetric RANS solver was coupled to an actuator disk model. This method was verified against solutions from the literature as well as three-dimensional simulations, and found to provide reasonable approximations to the turbine flowfield. Following this, a design methodology was developed with the goal of optimizing the shroud geometry and blade twist. In this procedure, rather than evaluating the forces based on a prescribed blade twist, the blade twist is described such that the angle of attack of each section is optimal with respect to the local flow angle. This procedure was used to obtain a design solution that exceeded the Betz limit by a factor of 2.645 based on rotor area and 1.43 based on the maximum shroud area. The optimal solution was evaluated using a three-dimensional RANS solver, suggesting the realizability of the design.

While structural considerations may limit the scalability of the turbine to very large configurations, this work suggests that shrouded turbines may be an effective solution at smaller scales.

Further development of shrouded turbines should consider additional features which have not been modeled in the present work. The turbine must be designed to tolerate gusts and mild crosswinds. Another important step is to incorporate structural analysis into the design of a shrouded turbine. With a structural model in place, the possibility of increasing the planform area towards the wind turbine tip can be addressed. This can have a significant impact on the power production of a shrouded turbine, as more torque can be generated for the same amount of thrust in the outboard portion of the blade.

\section{ACKNOWLEDGMENT}

The first author was supported by the DoD NDSEG fellowship.

\section{REFERENCES}

1. K. Abe, M. Nishida, A. Sakurai, Y. Ohya, H. Kihara, E. Wada, and K. Sato. Experimental and numerical investigations of flow fields behind a small wind turbine with a flanged diffuser. Journal of wind engineering and industrial aerodynamics, 93(12):951-970, 2005.

2. K. Abe and Y. Ohya. An investigation of flow fields around flanged diffusers using cfd. Journal of Wind Engineering and Industrial Aerodynamics, 92, 2004.

3. A. Aranake, V. Lakshminarayan, and K. Duraisamy. Assessment of transition model and cfd methodology for wind turbine flows. In $42^{\text {nd }}$ AIAA Fluid Dynamics Conference and Exhibit, New Orleans, LA, June 2012. 
4. A. Aranake, V. Lakshminarayan, and K. Duraisamy. Assessment of low-order theories for analysis and design of shrouded wind turbines using cfd. In Journal of Physics: Conference Series, volume 524, page 012077. IOP Publishing, 2014.

5. A. Aranake, V. Lakshminarayan, and K. Duraisamy. Computational analysis of shrouded wind turbine configurations using a 3-dimensional RANS solver. Renewable Energy, 75:818-832, 2015.

6. A. Aranake. Analysis and design of shrouded turbines. Doctoral Dissertation, Stanford University, 2015.

7. J. Baeder, K. Duraisamy, and V. Lakshminarayan. Rans predictions of complex hovering rotor configurations: From micro scale to full scale. Computational Fluid Dynamics Journal, 18(3-4), 2011.

8. R. Bontempo, M. Cardone, M. Manna, and G. Vorraro. Ducted propeller flow analysis by means of a generalized actuator disk model. Energy Procedia, 45:1107-1115, 2014.

9. K. Duraisamy, M. Ramasamy, J. Baeder, and G. Leishman. High resolution computational and experimental study of hovering rotor tip vortex formation. AIAA Journal, 45(11), 2007.

10. K. Duraisamy and J. Baeder. High resolution wake capturing methodology for hovering rotor simulations. Journal of the American Helicopter Society, 52(2), 2007.

11. K. Duraisamy and J. Baeder. Numerical simulation of the effects of spanwise blowing on wing-tip vortex formation and evolution. Journal of aircraft, 43(4):996-1006, 2006.

12. B. L. Gilbert and K. M. Foreman. Experiments with a diffuser-augmented model wind turbine. Journal of Energy Resources Technology, 105, 1983.

13. B. Gilbert, R. Oman, and K. Foreman. Fluid dynamics of diffuser-augmented wind turbines. Journal of Energy, 2(6):368-374, 1978.

14. P. E Gill, W. Murray, and M. Saunders. Users guide for SNOPT version 7: Software for large-scale nonlinear programming. 2006.

15. O. Igra. Research and development for shrouded wind turbines. Energy cons. \& Mgmt, 21:13-48, 1981.

16. P. Jamieson. Beating betz: Energy extraction limits in a constrained flow field. Journal of Solar Energy Engineering, $131,2009$.

17. V. K. Lakshminarayan. Computational investigation of micro-scale coaxial rotor aerodynamics in hover. Ph.D. dissertation, Department of Aerospace Engineering, 2009.

18. A. Lambe and J. Martins. Extensions to the design structure matrix for the description of multidisciplinary design, analysis, and optimization processes. Structural and Multidisciplinary Optimization, 46(2):273-284, 2012.

19. G. Lilley and W. Rainbird. A preliminary report on the design and performance of a ducted windmill, report 102. College of Aeronautics, 1956. 
20. J. Martins and A. Lambe. Multidisciplinary design optimization: a survey of architectures. AIAA journal, 51(9):2049-2075, 2013.

21. J. Mathews and K. D Fink. Numerical methods using MATLAB, volume 31. Prentice hall Upper Saddle River, NJ, 1999.

22. A. Ning and K. Dykes. Understanding the benefits and limitations of increasing maximum rotor tip speed for utilityscale wind turbines. In Journal of Physics: Conference Series, volume 524, page 012087, 2014.

23. A. Ning, R. Damiani, and P. Moriarty. Objectives and constraints for wind turbine optimization. Journal of Solar Energy Engineering, 2014.

24. S. Ning. A simple solution method for the blade element momentum equations with guaranteed convergence. Wind Energy, 17(9):1327-1345, 2014.

25. Y. Ohya and T. Karasudani. A shrouded wind turbine generating high output power with wind-lens technology. Energies, 131, 2010.

26. Y. Ohya, T. Karasudani, A. Sakurai, K. Abe, and M. Inoue. Development of a shrouded wind turbine with a flanged diffuser. Journal of wind engineering and industrial aerodynamics, 96(5):524-539, 2008.

27. D.G. Phillips, R. Flay, T. Nash. Aerodynamic analysis and monitoring of the vortec 7 diffuser-augmented wind turbine. 1999.

28. T. Pulliam and D. Chaussee. A diagonal form of an implicit approximate factorization algorithm. Journal of Computational Physics, 39(2):347-363, 1981.

29. R. R. Ramsay, J. M. Janiszewska, and G. M. Gregorek. Wind tunnel testing of three s809 aileron configurations for use on horizontal axis wind turbines : Airfoil performance report. The Ohio State University, (DE-AC3683CH10093), 1996.

30. H. Sobieczky. Parametric airfoils and wings. In Recent Development of Aerodynamic Design Methodologies, pages 71-87. Springer, 1999.

31. J. Sørensen, W. Shen, and X. Munduate. Analysis of wake states by a full-field actuator disc model. Wind Energy, 1(2):73-88, 1998.

32. G. van Bussel. The science of making more torque from wind: Diffuser experiments and theory revisited. Journal of Physics: Conference Series, 75, 2007.

33. M. J. Werle and W. M. Presz Jr. Ducted wind/water turbines and propellers revisited. Journal of Propulsion and Power, 24(5), 2008. 